OPEN ACCESS

Edited by:

Guoxiang Xie,

University of Hawaii Cancer Center

United States

Reviewed by:

Haiwei Gu,

Arizona State University, United States

Ru Yan,

University of Macau, Macau

*Correspondence:

Jinjun Shan

jshan@njucm.edu.cn

Liuqing $D i$

diliuqing928@163.com

tThese authors have contributed equally to this work

Specialty section: This article was submitted to Translational Pharmacology,

a section of the journal

Frontiers in Pharmacology

Received: 12 April 2018

Accepted: 23 July 2018

Published: 14 August 2018

Citation:

Shan J, Peng L, Qian W, Xie T, Kang A, Gao B and Di L (2018)

Integrated Serum and Fecal

Metabolomics Study of

Collagen-Induced Arthritis Rats and the Therapeutic Effects of the Zushima

Tablet. Front. Pharmacol. 9:891. doi: 10.3389/fphar.2018.00891

\section{Integrated Serum and Fecal Metabolomics Study of Collagen-Induced Arthritis Rats and the Therapeutic Effects of the Zushima Tablet}

\author{
Jinjun Shan 1,2*t, Linxiu Peng ${ }^{3 \dagger}$, Wenjuan Qian ${ }^{3}$, Tong Xie ${ }^{1,2}$, An Kang ${ }^{3,4}$, Bei Gao ${ }^{5}$ and \\ Liuqing $D i^{1,3,4 *}$
}

1 Jiangsu Key Laboratory of Pediatric Respiratory Disease, Institute of Pediatrics, Nanjing University of Chinese Medicine, Nanjing, China, ${ }^{2}$ Medical Metabolomics Center, Nanjing University of Chinese Medicine, Nanjing, China, ${ }^{3}$ State Key Laboratory Cultivation Base for TCM Quality and Efficacy, School of Pharmacy, Nanjing University of Chinese Medicine, Nanjing, China, ${ }^{4}$ Jiangsu Key Laboratory for Functional Substance of Chinese Medicine, Nanjing, China, ${ }^{5}$ Genome Center of UC Davis, NIH West Coast Metabolomics Center, Davis, CA, United States

The Zushima tablet (ZT) has been used for decades in the clinical treatment of rheumatoid arthritis (RA) in China. However, its therapeutic mechanism is unclear. In this study, we aimed to explore the distinctive metabolic patterns in collagen-induced arthritis (CIA) rats and evaluate the therapeutic effects of ZT on RA using untargeted serum and fecal metabolomics approaches based on gas chromatography coupled with mass spectrometry. Body weight, hind paw swelling, TNF- $\alpha$ and IL-1 $\beta$ levels, arthritis scores, and histopathological parameters were assessed. In the metabolomics study, 31 altered metabolites in the serum and 30 in the feces were identified by comparing the model with the control group using statistical processing. These altered metabolites revealed that the tricarboxylic acid cycle, glycolysis metabolism, fatty acid metabolism, and purine metabolism were disturbed in CIA rats, and most of these altered metabolites including I-isoleucine, I-aspartic acid, pyruvic acid, cholic acid, and hypoxanthine, were rectified by ZT. Furthermore, short-chain fatty acids in feces were quantitatively determined, and the results showed that ZT could regulate the levels of propionate, butyrate, and valerate in CIA rats. Then, gut microbiota were analyzed by $16 \mathrm{~S}$ rRNA analysis. Our results showed that Firmicutes and Bacteroidetes were the most abundant bacteria in rats. The levels of 19 types of bacteria at the family level were altered in RA rats, and most of them could be regulated by $Z \mathrm{ZT}$. This study demonstrated that metabolomics analysis is a powerful tool for providing novel insight into RA and for elucidating the potential mechanism of ZT.

Keywords: metabolomics, rheumatoid arthritis, Chinese medicine, gut microbiota, gas chromatography-mass spectrometry 


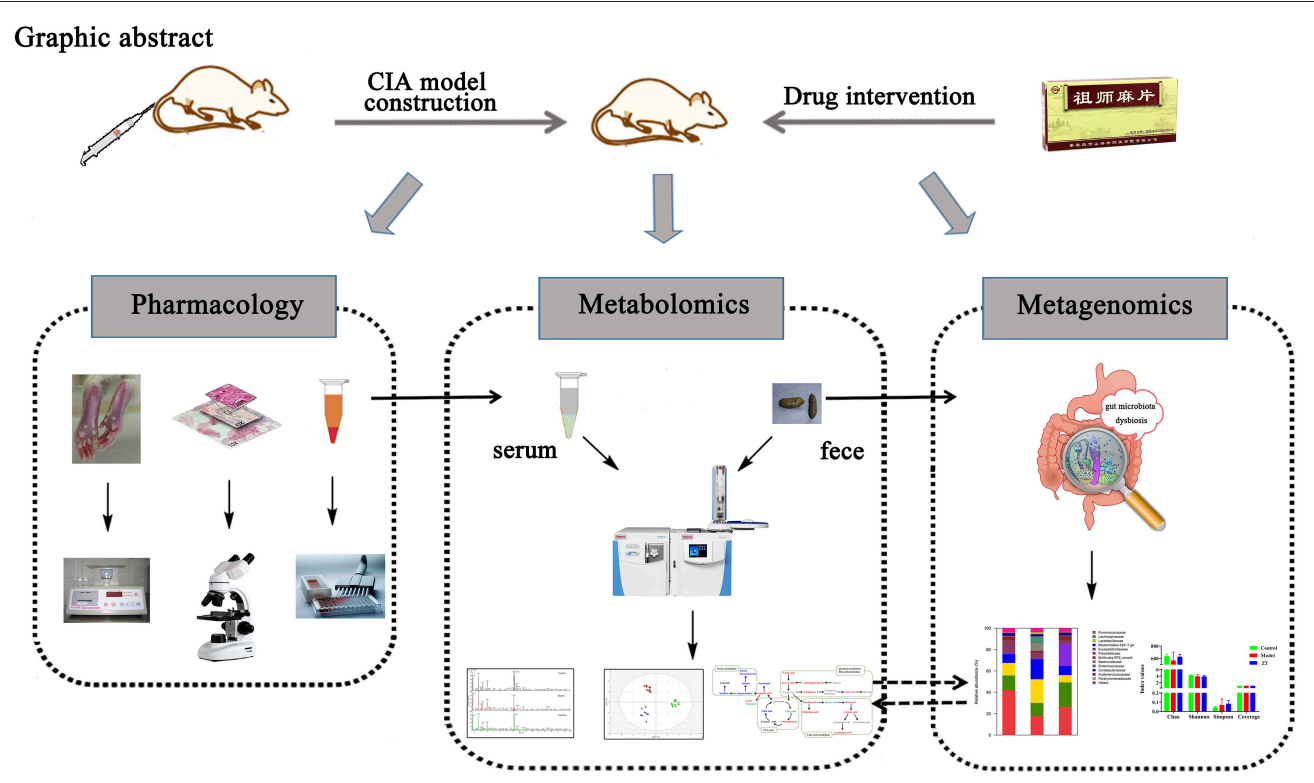

Graphical Abstract | Summary of experiment processes includes pharmacology, metabolomics and metagenomics.

\section{INTRODUCTION}

Rheumatoid arthritis (RA) is a chronic, systemic, autoimmune disease accompanied by persistent synovitis (inflammation of the synovial membrane), emergence of cartilage erosion, and destruction of the bone and joints (Qi et al., 2014). It has been reported that RA is always associated with oxidative stress (Kundu et al., 2012) and high levels of cytokines, including interleukins (IL-1) and tumor necrosis factor- $\alpha$ (TNF- $\alpha$ ) (Arend and Dayer, 1995). Although many studies have focused on RA, its pathogenesis is still not clear. In recent years, several studies have reported that impairment in the interaction between the gut microbiota and mucosal immune system can lead to inflammatory diseases such as RA. This finding contributed to a better understanding of this complex disease (Cai et al., 2012; Gomez et al., 2012; Li et al., 2015a; Chen et al., 2016).

Owing to its pathogenetic holism and complexity, no specific medicine has been able to effectively cure RA. Diseasemodifying anti-rheumatic drugs (DMARDs) and non-steroidal anti-inflammatory drugs (NSAIDs) have improved clinical symptoms in patients with RA (Van Vollenhoven, 2009). However, side effects such as liver and gastrointestinal disorders limit their extensive clinical use (Cavagna et al., 2013; Moller et al., 2015). Herbal medicines, which are widely used in some traditional medical systems, have provided a huge source of new drugs that can be used to treat complicated diseases ( $\mathrm{Li}$ et al., 2015b).

The Zushima tablet (ZT), a traditional Chinese patented prescription medicine, was officially recorded in the Pharmacopeia of the People's Republic of China (2015 edition) for the treatment of RA. ZT mainly contains coumarins and flavonoids, such as daphnetin, daphnoretin, 7-hydroxycoumarin, yuankanin, daphneticin, and rutarensin. These components were reported to have analgesic, anti-inflammatory, and anti-bacterial activities (Gao et al., 2008; Chen et al., 2011; Huyiligeqi et al., 2016). However, the therapeutic effects of ZT on RA should be investigated further to understand its comprehensive mechanism of action for better clinical application.

Metabolomics is usually applied to understand the function of biological systems based on the global metabolite profiles influenced by pathological stimuli or drug treatments in biological samples (Gu et al., 2015). Generally, serum and fecal metabolomics provide biomarker information associated with physiological or pathophysiological processes (Huyiligeqi et al., 2016; Khamis et al., 2017). Therefore, the combination of the two metabolomics approaches can provide a more comprehensive and detailed holistic metabolic profiling. In this study, serum and fecal metabolomics approaches were carried out using gas chromatography-mass spectrometry (GC-MS) to obtain the metabolites altered by RA and elucidate the regulatory mechanism of ZT. Furthermore, fecal samples were investigated by $16 \mathrm{~S}$ rRNA sequence analysis to analyze the altered gut microbiota and subsequently explore the association of gut microbiota with endogenous metabolites. The present study aimed to investigate the pathogenesis of RA through global serum and fecal metabolomics approaches, which can aid in the identification of metabolites associated with gut microbiota, and to evaluate the potential protective mechanism of ZT.

\section{MATERIALS AND METHODS}

\section{Chemicals and Reagents}

Bovine type II collagen was purchased from Chondrex (Redmond, WA, USA). Acetic acid was purchased from 


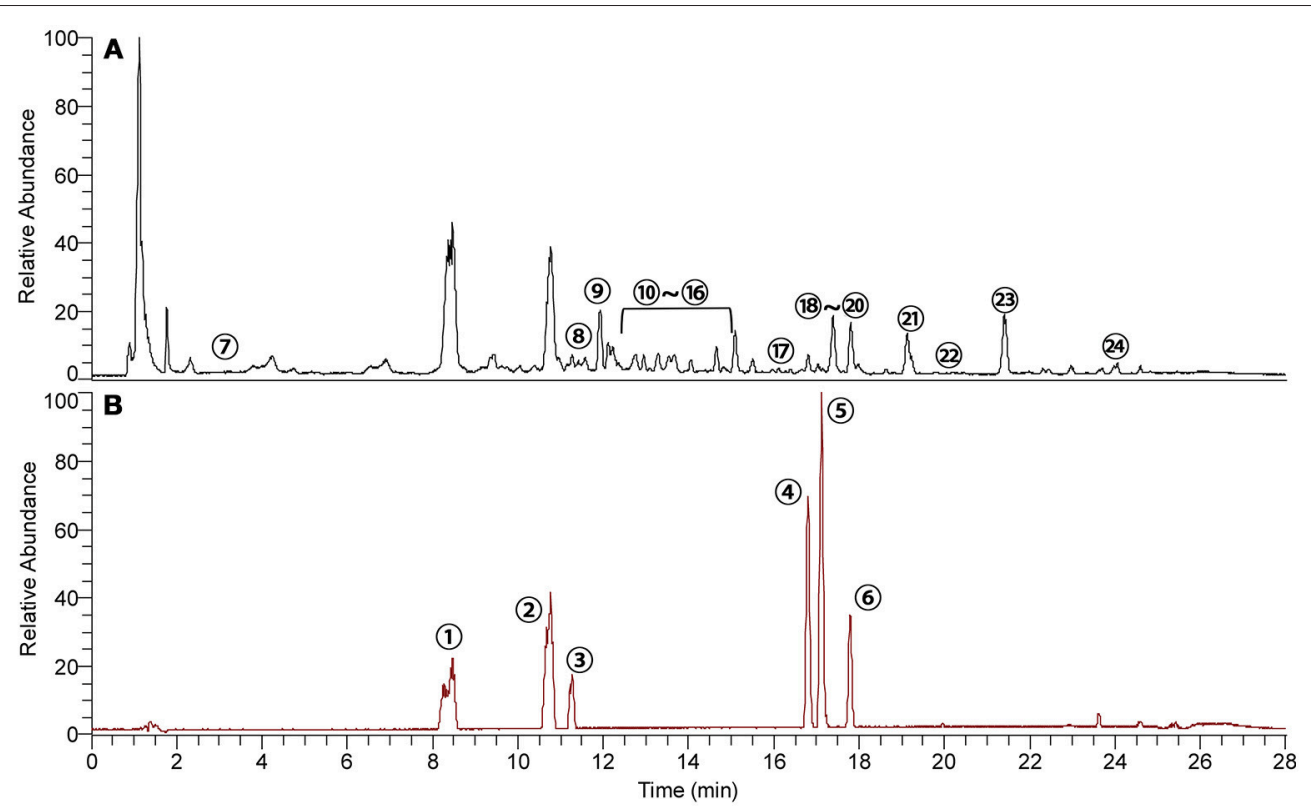

FIGURE 1 | Typical base peak intensity chromatograms of (A) ZT extract and (B) a six-standard mixture analyzed by UHPLC-Q Exactive-Orbitrap-MS. The components are listed in Table 1.

Nanjing Chemical Reagents Co., LTD (Nanjing, Jiangsu, China). Freund's complete/incomplete adjuvant, methoxyamine hydrochloride, pyridine, $1,2-{ }^{13} \mathrm{C}$-myristic acid, and $\mathrm{N}$, OBis(trimethylsilyl)trifluoroacetamide with trimethylchlorosilane (BSTFA) were all obtained from Sigma-Aldrich (St. Louis, MO, USA). Both n-hexane and acetone were purchased from ROE Scientific (St. Louis, MO, USA). Methanol of MS grade was supplied by Merck Millipore (Billerica, MA, USA). ZT was purchased from Shanhaiguan Pharmaceutical Co., Ltd (Qinhuangdao, Hebei, China, batch number: 20161202). The quality of ZT was also evaluated and the method is shown in the Supplementary File. Carboxymethylcellulose sodium (CMC$\mathrm{Na}$; 300-800 mPa.S) was obtained from Sinopharm Chemical Reagent Co., Ltd (Shanghai, China). Distilled water was purified using a Milli-Q purification system (Millipore, Milford, MA, USA).

\section{Animals}

Thirty-four male Sprague-Dawley rats weighing 180-220 g were supplied by Jiesijie lab animal Ltd. (Shanghai, China), and the animal certification number was SCXK-2013-0006. All rats were fed in an environmentally controlled room with constant temperature $\left(20-24{ }^{\circ} \mathrm{C}\right.$ ) and humidity (45-60\%) of SPF grade with a standard $12 \mathrm{~h}$ light/dark cycle for 5 days before the formal experiment. After 5 days of adaptive feeding with free access to water and chow during the experiment, the rats were divided into two groups: 10 rats in the control group and 24 rats for collagen-induced arthritis (CIA) model development. The animal experiments were performed under the guidelines of the Animal Ethics Committee of Nanjing University of Chinese Medicine.

\section{CIA Model Construction}

The CIA rat model was established according to the Experimental Methodology of Pharmacology ( Wu and Li, 2010). Bovine type II collagen $(20 \mathrm{mg})$ was dissolved in $10 \mathrm{~mL}$ acetic acid solution $(0.05 \mathrm{M})$ and the mixture (CII-AA) was shaken at $4^{\circ} \mathrm{C}$ overnight. After $12 \mathrm{~h}$, CII-AA was emulsified with Complete Freund's Adjuvant (CFA) at a ratio of 1:1 using an Ultra-Turrax high speed disperser (IKA ${ }^{\circledR}$ Works Guangzhou, Guangzhou, Guangdong, China) until the CII-CFA emulsion remained unscattered in water. In addition, CII-AA was emulsified with Incomplete Freund's Adjuvant (IFA) to prepare the CII-IFA emulsion using the same protocol mentioned above.

CIA model rats were intradermally (i.d.) injected with $0.2 \mathrm{~mL}$ CII-CFA emulsion at the base of the tail (day 1). On day 7 after the primary immunization, the rats were boosted intradermally with $0.1 \mathrm{~mL}$ CII-IFA. The normal rats were injected with the same volume of physiological saline.

\section{Drug Administration and Sample Collection}

Almost 2 weeks after model development, the rat models with RA were divided randomly into model and ZT groups. To assess its therapeutic efficacy, ZT was suspended in $0.5 \%$ CMC$\mathrm{Na}$ solution, and rats in the ZT group were administered ZT intragastrically $(0.6 \mathrm{~g} / \mathrm{kg}$, each tablet weighs $0.3 \mathrm{~g}, 2$-fold the clinical dosage) from the day after the onset of arthritis (day 14) and once daily until day 36 of the experiment. In addition, the rats in the control and model groups were treated with the same volume $(10 \mathrm{~mL} / \mathrm{kg})$ of $0.5 \% \mathrm{CMC}-\mathrm{Na}$ solution.

Serum and fecal samples were collected on day 0 (prior to immunization), 14 (prior to therapy), 21, and 36 , respectively. 
TABLE 1 | The chemical components identified in the Zushima tablet.

\begin{tabular}{|c|c|c|c|c|c|c|}
\hline No. & Compound & RT(min) & $\mathrm{m} / \mathbf{z}$ & Formula & Delta ppm & MS2 \\
\hline 1 & Daphnetin & 8.45 & 177.01845 & $\mathrm{C} 9 \mathrm{H} 5 \mathrm{O} 4$ & 0.275 & 121.03 \\
\hline 2 & 7-Hydroxycoumarin & 10.77 & 161.02341 & $\mathrm{C9H} 5 \mathrm{O} 3$ & 0.179 & 133.03 \\
\hline 3 & Luteoloside & 11.27 & 447.09354 & $\mathrm{C} 21 \mathrm{H} 19011$ & 1.932 & 285.04 \\
\hline 4 & Apigenin & 16.78 & 269.04556 & C15H9O5 & 1.05 & 117.03 \\
\hline 5 & Kaempferol & 17.11 & 285.04037 & C15H9O6 & 1.196 & \\
\hline 6 & Daphnoretin & 17.77 & 351.05011 & $\mathrm{C} 19 \mathrm{H} 11 \mathrm{O} 7$ & 0.181 & $163.00,191.00,336.03$ \\
\hline 7 & $\begin{array}{l}\text { Daphnetin-8-O- } \beta \text {-D-glucopyranosyl- } \\
\text { (1-6)- } \beta \text {-D-glucopyranoside }\end{array}$ & 3.76 & 501.1246 & $\mathrm{C} 21 \mathrm{H} 25 \mathrm{O} 14$ & 0.718 & 176.94 \\
\hline 8 & Daphjamilin & $11.24,12.72$ & 467.09799 & $\mathrm{C} 24 \mathrm{H} 19010$ & 0.717 & $277.05,321.04$ \\
\hline 9 & Giarldoid B & 11.91 & 515.08264 & $\mathrm{C} 24 \mathrm{H} 19013$ & 0.623 & $177.02,339.07$ \\
\hline 10 & Yuankanin & 12.28 & 577.15619 & $\mathrm{C} 27 \mathrm{H} 29014$ & 1.008 & $268.04,283.06$ \\
\hline 11 & $\begin{array}{l}\text { (-)-pinoresinol-4-O- } \beta-\mathrm{D}- \\
\text { glucopyranoside }\end{array}$ & 13.11 & 519.18549 & $\mathrm{C} 26 \mathrm{H} 31011$ & -0.598 & $83.01,357.03$ \\
\hline 12 & Daphgilin & 13.53 & 353.02969 & $\mathrm{C} 18 \mathrm{H} 9 \mathrm{O} 8$ & 0.496 & 177.01855 \\
\hline 13 & genkwanin-5-O- $\beta$-D-glucoside & 13.03 & 445.11377 & $\mathrm{C} 22 \mathrm{H} 21010$ & 0.847 & 268.04, 283.06 \\
\hline 14 & vladinoID & 13.27 & 373.12851 & $\mathrm{C} 2 \mathrm{OH} 21 \mathrm{O} 7$ & 0.331 & $298.08,313.11$ \\
\hline 15 & Rutarensin & 14.67 & 657.1449 & C31H29O16 & -0.111 & $351.08,393.16$ \\
\hline 16 & Luteolin & 15.09 & 285.04074 & $\mathrm{C} 15 \mathrm{H} 9 \mathrm{O} 6$ & 1.376 & 133.03 \\
\hline 17 & Daphnodorin A/C/D1/D2 & $16.11 / 16.39 / 18.00$ & 525.11841 & $\mathrm{C} 30 \mathrm{H} 21 \mathrm{O} 9$ & 0.401 & $119.05,151.00,431.08$ \\
\hline 18 & Daphneticin & 16.29 & 385.09341 & $\mathrm{C} 2 \mathrm{OH} 17 \mathrm{O} 8$ & 0.276 & $175.93,209.09,121.03$ \\
\hline 19 & (-)-pinoresinol & 17.05 & 357.13474 & $\mathrm{C} 2 \mathrm{OH} 21 \mathrm{O} 6$ & 0.465 & $83.01,122.04,137.06,221.08$ \\
\hline 20 & (-)-Dihydrosesamin & 17.18 & 355.1192 & $\mathrm{C} 2 \mathrm{OH} 1906$ & 0.455 & $160.05,176.01,219.07$ \\
\hline 21 & Daphneolone & 19.11 & 269.11874 & $\mathrm{C} 17 \mathrm{H} 17 \mathrm{O} 3$ & 0.549 & $93.03,135.04$ \\
\hline 22 & 12-hydroxydaphnetoxin & 20.21 & 497.18298 & $\mathrm{C} 27 \mathrm{H} 2909$ & 0.541 & $121.03,327.12$ \\
\hline 23 & Genkwanin & 21.41 & 283.06119 & $\mathrm{C} 16 \mathrm{H} 11 \mathrm{O} 5$ & 1.06 & 268.04 \\
\hline 24 & Daphnetoxin & 23.91 & 481.18756 & $\mathrm{C} 27 \mathrm{H} 2908$ & 0.986 & 121.03 \\
\hline
\end{tabular}

Blood samples were collected from the postorbital venous plexus veins and kept at room temperature for $1 \mathrm{~h}$. Then, the samples were centrifuged at 4,000 rpm for $5 \mathrm{~min}$ to obtain the serum. In addition, around 2-3 stool samples were stored in liquid nitrogen immediately after collection. Both serum and feces were stored at $-80^{\circ} \mathrm{C}$ until analysis.

\section{Basic Physiological Parameters}

During the experiment, three basic physical parameters were selected for evaluating model development and efficacy of ZT: body weight, paw swelling, and arthritis scores. The body weights and paw swelling were assessed every 3-4 days after the rats were injected with collagen. As a significant index for evaluating the seriousness of RA, paw swelling was expressed using the average value of left and right hind paws. Meanwhile, the arthritic scores were recorded from day 12 to 36 at an interval of 4 days. The severity of arthritis was graded according to the following criteria (Zhang et al., 2014): 0 score: no pathological changes; 1 score: slight redness or swelling in the palms; 2 score: slight redness or swelling in the ankle joint and toe joints; 3 score: moderate redness and swelling in the palms, ankle, and toe joints; and 4 score: serious redness and swelling in the palms, ankle, and toe joints. All four paws were assessed, and the values were added for each rat. The highest score for each rat was 16.

\section{Quantification of Serum TNF- $\alpha$ and IL-1 $\beta$ and histopathology Analysis}

All rats were sacrificed on day 36 , and blood was collected to obtain serum for measuring the inflammatory factors TNF- $\alpha$ and IL-1 $\beta$ using ELISA kits (Multi Sciences, Hangzhou, Zhejiang, China). Meanwhile, the right ankle joint of each rat was fixed in formalin for histopathologic analysis.

\section{Serum and Fecal Sample Preparation for Metabolomics Analysis}

Serum samples were thawed at $4^{\circ} \mathrm{C}$, and an aliquot of $50 \mu \mathrm{L}$ serum was transferred into a $1.5 \mathrm{~mL}$ Eppendorf tube. Then, 200 $\mu \mathrm{L}$ methanol (with $12.5 \mu \mathrm{g} / \mathrm{mL} 1,2-{ }^{13} \mathrm{C}$-myristic acid) was added to precipitate proteins as well as to mix the internal standards with samples. The samples were centrifuged at 14,000 rpm for 10 min with an Allegra 64R High-Speed Refrigerated Centrifuge (Beckman Coulter, Brea, CA, USA) after vortexing for $3 \mathrm{~min}$ using a vortex-genie (Scientific Industries, Bohemia, NY, USA). The supernatant $(100 \mu \mathrm{L})$ was transferred into a new tube and evaporated to dryness at $45^{\circ} \mathrm{C}$ and $15 \mathrm{kPa}$ in a Thermo SPD1010230 SpeedVac Concentrator (Thermo Fisher, San Jose, CA, USA). After drying, $30 \mu \mathrm{L}$ of methoxyamine hydrochloride dissolved in pyridine $(\mathrm{w} / \mathrm{v}, 10 \mathrm{mg} / \mathrm{mL}$ ) was added and vortexed for $5 \mathrm{~min}$. Then, the samples were shaken at a speed of $300 \mathrm{rpm}$ for $90 \mathrm{~min}$ 

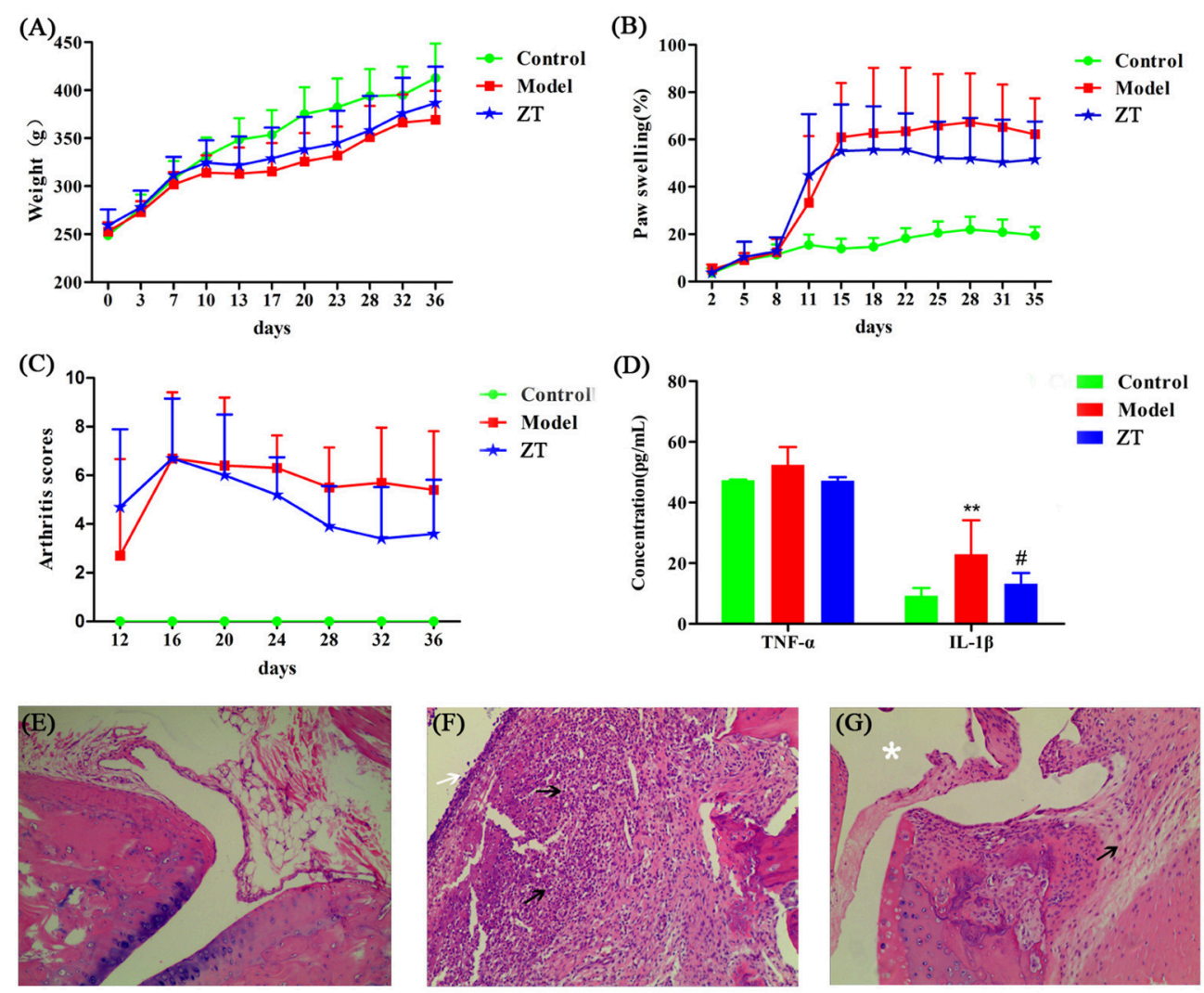

FIGURE 2 | Evaluation of CIA model construction and efficacy of ZT. (A) Body weight, (B) paw swelling, (C) arthritis score, and (D) serum levels of inflammatory factors TNF- $\alpha$ and IL-1 $\beta$. All the above values are expressed as Mean \pm SD $(n=10)$, ${ }^{*}$, vs. Control, $P<0.01$; \#, vs. Model, $P<0.05$. Representative photographs of histological examination of synovial tissues in the ankle joint (100 $\times$ magnification). (E) Control group, (F) model group, (G) ZT group. The black arrows indicate numbers of inflammatory cells, such as plasma cells, lymphocytes, and neutrophils, the white arrow indicate destoryed cartilage. The asterisks indicate clear joint cavity.

in a thermostatic oscillator at $30^{\circ} \mathrm{C}$. Subsequently, $30 \mu \mathrm{L}$ of BSTFA (1\% TMS) was added and shaken for another $30 \mathrm{~min}$ at $37^{\circ} \mathrm{C}$.

About $50 \mathrm{mg}$ of feces was spiked with $0.5 \mathrm{~mL}$ water and homogenized for $5 \mathrm{~min}$, and the mixtures were centrifuged at $13,000 \mathrm{rpm}$ for $10 \mathrm{~min}$ to obtain $0.4 \mathrm{~mL}$ water supernatant. In addition, $0.5 \mathrm{~mL}$ methanol was added to the residue and homogenized once again. Next, $0.4 \mathrm{~mL}$ methanol supernatant was spiked with the water supernatant. After vortexing of the supernatant from the above steps for $10 \mathrm{~s}, 0.4 \mathrm{~mL}$ of fecal extract was transferred into a new tube with $10 \mu \mathrm{L}$ of $1,2-{ }^{13} \mathrm{C}$-myristic acid $(400 \mu \mathrm{g} / \mathrm{mL})$, and then the samples were dried. A slight difference was exhibited for fecal samples when compared to serum samples in terms of derivatization. The volume of both methoxyamine hydrochloride pyridine solution and BSTFA was $60 \mu \mathrm{L}$, and the mixture was shaken for $60 \mathrm{~min}$ after adding BSTFA.

After derivatization, the mixture was centrifuged at $18,000 \mathrm{rpm}$ for $10 \mathrm{~min}$ before injecting into the GC-MS. All derivatization must be performed in a waterless environment. Quality control (QC) samples of serum and feces were also prepared and derivatized according to the above protocol, and they were run after every 10 samples to monitor retention time and elution order of metabolites.

\section{GC-MS Conditions for Metabolomics Analysis}

The metabolomics analysis of serum and fecal samples was performed using the Trace 1310-TSQ 8000 Evo (Thermo Fisher, San Jose, CA, USA). The analytes were separated on a TG-5MS capillary column $(0.25 \mathrm{~mm} \times 30 \mathrm{~m} \times 0.25 \mu \mathrm{m}$, Thermo Fisher, San Jose, CA, USA) with a split ratio of 20:1. The injection volume was $1 \mu \mathrm{L}$. The gradient heating program was conducted as follows: $0-1 \mathrm{~min}, 60^{\circ} \mathrm{C}$; $1-14 \mathrm{~min}, 60-320^{\circ} \mathrm{C}$; $14-19 \mathrm{~min}, 320^{\circ} \mathrm{C}$. Helium (99.999\%) was used as the carrier gas, with a flow rate of $1.2 \mathrm{~mL} / \mathrm{min}$. The TSQ 8000 was equipped with an Electron Ionization (EI) source. The ionization energy was $70 \mathrm{eV}$, the source temperature was $280^{\circ} \mathrm{C}$, and the transfer line was held at $290^{\circ} \mathrm{C}$. The GC-MS data were acquired after a solvent delay of $3.65 \mathrm{~min}$, and the MS scan range was $50-500 \mathrm{~m} / \mathrm{z}$.

QC was assured by (a) randomization of sequence, (b) injection of QC samples between every 10 actual samples, and (c) checking the peak shape and intensity of the spiked internal standard $\left(1,2-{ }^{13} \mathrm{C}\right.$-myristic acid). 
TABLE 2 | Significant serum metabolites associated with rheumatoid arthritis based on gas chromatography-mass spectrometry.

\begin{tabular}{|c|c|c|c|c|c|c|}
\hline No. & Match & $\mathbf{R T}(\min )$ & Quant mass (m/z) & Day & ANOVA & $q$-test \\
\hline 1 & Allantoic acid & $10.05,10.93$ & 331,259 & 14 & $0.011^{b}$ & $0.027^{b}$ \\
\hline 2 & Scyllitol & 10.45 & 318 & 14 & $<0.001^{b}$ & $<0.001^{b}$ \\
\hline 3 & Vitamin e & 15.29 & 237 & 21 & $0.015^{c}$ & $0.021^{\mathrm{C}}$ \\
\hline 4 & Arachidonic acid & 12.21 & 73 & 14 & $0.031^{b}$ & $0.012^{b}$ \\
\hline 5 & L-Asparagine & $7.79,8.38,8.81$ & $84,116,73$ & 21 & $<0.001^{\mathrm{C}}$ & $0.013^{C}$ \\
\hline 6 & L-Aspartic acid* & $7.91,7.28$ & 232,73 & 14 & $<0.001^{\mathrm{b}}$ & $<0.001^{b}$ \\
\hline 7 & Cholesterol & 15.46 & 129 & 21 & $0.003^{C}$ & $0.006^{C}$ \\
\hline 8 & Cholic acid & 16.28 & 253 & 36 & $0.001^{d}$ & $0.002^{d}$ \\
\hline 9 & Citric acid* & 9.67 & 273 & 14 & $<0.001^{\mathrm{b}}$ & $<0.001^{\mathrm{b}}$ \\
\hline 10 & D-Galactose & 10.15 & 73 & 14 & $0.027^{b}$ & $0.015^{\mathrm{b}}$ \\
\hline 11 & Glucose 6-phosphate & 12.15 & 299 & 14 & $0.01^{\mathrm{b}}$ & $0.018^{\mathrm{b}}$ \\
\hline 12 & Glycine & $5.07,6.51$ & 102,174 & 14 & $0.008^{b}$ & $0.002^{b}$ \\
\hline 13 & Glycolic acid & 4.73 & 147 & 21 & $0.014^{\mathrm{C}}$ & $0.004^{\mathrm{C}}$ \\
\hline 14 & L-Isoleucine* & 6.41 & 158 & 14,21 & $0.003^{b}, 0.006^{c}$ & $0.001^{b}, 0.029^{c}$ \\
\hline 15 & L-Lactic acid & 4.61 & 207 & 14 & $0.023^{b}$ & $0.028^{b}$ \\
\hline 16 & Linoleic acid* & 11.51 & 75 & 0,21 & $0.04^{\mathrm{a}},<0.001^{\mathrm{c}}$ & $0.03^{a},<0.001^{c}$ \\
\hline 17 & L-Lysine* & 10.19 & 174 & 14 & $0.037^{\mathrm{b}}$ & $0.011^{\mathrm{b}}$ \\
\hline 18 & D-Mannose & 10.12 & 87 & 14,36 & $0.008^{b}, 0.034^{d}$ & $0.015 b, 0.01^{d}$ \\
\hline 19 & Galactaric acid & 10.69 & 333 & 14 & $0.009^{b}$ & $0.006^{\mathrm{b}}$ \\
\hline 20 & Oleic acid & 11.53 & 339 & 0,21 & $0.013^{a}, 0.006^{c}$ & $0.008^{a}, 0.008^{c}$ \\
\hline 21 & O-Phosphoethanolamine & 9.48 & 299 & 14 & $0.012^{\mathrm{b}}$ & $0.025^{\mathrm{b}}$ \\
\hline 22 & Palmitoleic acid & 10.63 & 311 & $0,14,36$ & $0.04^{a}, 0.014^{b}, 0.003^{d}$ & $0.017^{a}, 0.011^{b}, 0.002^{d}$ \\
\hline 23 & L-Phenylalanine* & $8.11,8.59$ & 120,73 & 14 & $0.004^{b}$ & $0.001^{\mathrm{b}}$ \\
\hline 24 & L-Proline* & 6.45 & 142 & 14,21 & $0.006^{b}, 0.014^{c}$ & $0.017^{b}, 0.016^{c}$ \\
\hline 25 & Pyruvic acid & 4.58 & 174 & 21 & $0.021^{\mathrm{c}}$ & $0.04^{\mathrm{C}}$ \\
\hline 26 & Spermidine & 11.8 & 144 & 36 & $<0.001^{d}$ & $<0.001^{d}$ \\
\hline 27 & Threonic acid & 8.19 & 73 & 36 & $0.014^{d}$ & $0.011^{d}$ \\
\hline 28 & 4-Hydroxyproline & $9.41,7.96$ & 274,230 & 14 & $0.045^{b}$ & $0.045^{b}$ \\
\hline 29 & L-Tyrosine & $10.01,10.28$ & 179,218 & 14 & $0.011^{b}$ & $0.003^{\mathrm{b}}$ \\
\hline 30 & Uracil & 6.72 & 241 & 14,21 & $<0.001^{b}, 0.005^{c}$ & $<0.001^{b}, 0.001^{c}$ \\
\hline 31 & 2-Deoxyglucose & 9.29 & 205 & 14 & $0.036^{b}$ & $0.028^{b}$ \\
\hline
\end{tabular}

${ }^{a} 0$ day; ${ }^{b} 14$ days; ${ }^{c} 21$ days; ${ }^{d} 36$ days; * confirmed with pure standards.

\section{Quantification of Short-Chain Fatty Acids (SCFAs) in Rat Feces}

The SCFAs including acetate, propionate, butyrate, isobutyrate, valerate, and isovalerate were bacterial metabolites produced by the fermentation of dietary fiber and resistant starches with the effect of specific colonic anaerobic bacteria (Tan et al., 2014). SCFAs (butyrate in particular) are the major and preferred metabolic substrate for colonocytes, providing energy and playing a key role in promoting the integrity of the colon (Suzuki et al., 2008). Therefore, different concentrations of SCFAs could indicate changes in intestinal function. The fecal samples at day 36 were selected and extracted to determine the content of SCFAs. The detailed extraction protocol and GC-MS conditions are described in the Supplementary File.

\section{S rRNA-Assisted High-Throughput Sequencing Analysis}

According to the fecal metabolomics and SCFAs results, RA might lead to alterations in the gut microbiota. Determination of gut microbiota differences between the control and model groups and whether ZT could normalize the changes in microbiota were critical aspects that needed to be explored. Thus, fecal samples from different groups were collected on day 36 and used for $16 \mathrm{~S}$ rRNA gene analysis, including extraction of genome DNA, amplicon generation, PCR products quantification and qualification, PCR products mixing and purification, and library preparation and sequencing.

Total DNA from samples was extracted using the E.Z.N.A.Stool DNA kit, and the DNA concentration and purity were monitored on $1 \%$ agarose gels. Briefly, the V4-V5 domain of the 16S rRNA gene was amplified using primers 515F (5'-GTGCCAGCMGCCGCGG-3') and 907R (5'-CCGTCAATTCMTTTRAGTTT-3'). PCR amplification was carried out in $30 \mu \mathrm{L}$ reaction system containing $15 \mu \mathrm{L}$ of Phusion ${ }^{\circledR}$ High-Fidelity PCR Master Mix (New England Biolabs), $0.2 \mu \mathrm{M}$ of forward and reverse primers, and about $10 \mathrm{ng}$ template DNA. Thermal cycling consisted of initial denaturation at $98^{\circ} \mathrm{C}$ for $1 \mathrm{~min}$ followed by 30 cycles of denaturation at $98^{\circ} \mathrm{C}$ for $10 \mathrm{~s}$, annealing at $50^{\circ} \mathrm{C}$ for $30 \mathrm{~s}$, elongation at $72^{\circ} \mathrm{C}$ for $60 \mathrm{~s}$, and then a single final extension step at $72^{\circ} \mathrm{C}$ for $5 \mathrm{~min}$. Then, mixed PCR products were purified with the GeneJET Gel Extraction 
TABLE 3 | Significant fecal metabolites associated with rheumatoid arthritis based on gas chromatography-mass spectrometry.

\begin{tabular}{|c|c|c|c|c|c|c|}
\hline No. & Biomarkers & RT(min) & Quant mass (m/z) & Day & ANOVA & $q$-test \\
\hline 1 & 1,5-anhydroglucitol & 9.84 & 73 & 21 & $0.007^{c}$ & $0.012^{C}$ \\
\hline 2 & 3-(3-Hydroxyphenyl)-propanoic acid & 9.29 & 310 & 21 & $0.043^{C}$ & $0.02^{\mathrm{C}}$ \\
\hline 3 & 4-Oxoproline & 7.95 & 156 & 21 & $0.008^{\mathrm{C}}$ & $0.046^{C}$ \\
\hline 4 & 5-hydroxy-3-indoleacetic acid & 11.49 & 290 & 21 & $0.033^{\mathrm{C}}$ & $0.026^{C}$ \\
\hline 5 & Adenine & 9.93 & 264 & 36 & $0.013^{d}$ & $0.006^{d}$ \\
\hline 6 & Beta-Alanine & 7.32 & 248 & 21 & $0.029^{C}$ & $0.009^{C}$ \\
\hline 7 & Citric acid* & 9.66 & 273 & 21 & $0.003^{C}$ & $0.001^{\mathrm{C}}$ \\
\hline 8 & Ethanolamine & 6.22 & 174 & 36 & $0.004^{d}$ & $0.001^{d}$ \\
\hline 9 & Gentisic acid & 9.4 & 355 & 21 & $0.001^{c}$ & $0.001^{c}$ \\
\hline 10 & Glycerol 3-phosphate & 9.35 & 299 & 21 & $0.004^{C}$ & $0.001^{\mathrm{C}}$ \\
\hline 11 & Heptadecanoic acid & 11.63 & 315 & 21 & $0.019^{C}$ & $0.007^{\mathrm{C}}$ \\
\hline 12 & Hypoxanthine & 9.59 & 265 & 21 & $0.005^{\mathrm{C}}$ & $0.003^{C}$ \\
\hline 13 & Inosine & 13.09 & 281 & 14 & $0.049^{b}$ & $0.018^{b}$ \\
\hline 14 & L-Glutamine & 9.39 & 73 & 36 & $0.007^{d}$ & $0.005^{d}$ \\
\hline 15 & L-Isoleucine* & 6.41 & 158 & 36 & $0.004^{d}$ & $0.001^{d}$ \\
\hline 16 & L-Leucine* & 6.25 & 158 & 36 & $0.006^{d}$ & $0.005^{d}$ \\
\hline 17 & L-Malic acid & 7.7 & 73 & 14 & $0.041^{b}$ & $0.013^{b}$ \\
\hline 18 & L-Phenylalanine* & $8.11,8.59$ & 120,73 & 36 & $0.025^{d}$ & $0.026^{d}$ \\
\hline 19 & L-Serine* & $6.13,6.87$ & 73,73 & 36 & $0.008^{d}$ & $0.003^{d}$ \\
\hline 20 & L-Tyrosine & 10.28 & 280 & 36 & $0.005^{d}$ & $0.005^{d}$ \\
\hline 21 & m-Cresol & 5.34 & 165 & 21 & $<0.001^{\mathrm{C}}$ & $0.005^{\mathrm{C}}$ \\
\hline 22 & Ornithine & $8.48,8.9,9.65$ & $142,328,73$ & 21 & $0.002^{c}$ & $0.024^{C}$ \\
\hline 23 & Pectin & 8.8 & 73 & 21 & $0.022^{C}$ & $0.006^{C}$ \\
\hline 24 & Phosphate & 6.26 & 299 & 21 & $0.004^{\mathrm{C}}$ & $0.001^{\mathrm{C}}$ \\
\hline 25 & Stearic acid & 11.62 & 341 & 21 & $0.013^{C}$ & $0.02^{\mathrm{C}}$ \\
\hline 26 & Thymine & 7.15 & 255 & 36 & $0.034^{d}$ & $0.013^{d}$ \\
\hline 27 & Ferulic acid & 11 & 323 & 36 & $0.043^{d}$ & $0.015^{d}$ \\
\hline 28 & Uracil & 6.72 & 241 & 21 & $0.019^{c}$ & $0.008^{C}$ \\
\hline 29 & Urea & 5.94 & 147 & 0,36 & $0.011^{a}, 0.037^{d}$ & $0.004^{a}, 0.006^{d}$ \\
\hline 30 & Xanthine & 10.69 & 353 & 21 & $0.013^{C}$ & $0.007^{\mathrm{C}}$ \\
\hline
\end{tabular}

${ }^{a} 0$ day; ${ }^{b} 14$ days; ${ }^{c} 21$ day; ${ }^{d} 36$ days; ${ }^{*}$ confirmed with pure standards.

Kit (Thermo Scientific). At last, the library was sequenced on an Illumina MiSeq platform, and 250 bp paired-end reads were generated. Finally, a total of 1,376,064 reads were obtained for subsequent analysis.

\section{Data Processing and Statistical Analysis}

GC-MS data (raw data files) of all samples were converted to ABF format using the ABF Converter (http://www.reifycs.com/ AbfConverter/; Cajka et al., 2017). All the data were imported into the MS-DIAL (v.2.7.2) software program (Tsugawa et al., 2015) for peak detection, identification, and alignment using the following parameters: retention time beginning, $3.65 \mathrm{~min}$; retention time end, 19 min; mass range beginning, $50 \mathrm{Da}$; mass range end, $500 \mathrm{Da}$; smoothing level, 3 scan; average peak width, 10 scan; retention index tolerance, 100,$000 ; \mathrm{m} / \mathrm{z}$ tolerance, 0.5 $\mathrm{Da}$; retention time tolerance for alignment, $0.3 \mathrm{~min}$. Metabolite identification was performed by comparing MS spectra and Ankle RI index with those of the National Institute of Standards and Technology (NIST), and the metabolites were confirmed only when the EI similarity was above $80 \%$. The alignment results were exported to a txt file labeled with the sample name, metabolites, peak height, and other information including retention time and quantification mass. Normalization is an important and effective method to exclude or reduce the unwanted overall variations in spectral data (Xi et al., 2014). In our research, data were normalized using sum normalization with MetaboAnalyst 4.0 (http://www.metaboanalyst.ca/) before statistical analysis. To search for changed metabolites in RA, data of normal distribution were obtained by log transformation. ANOVA and $q$-test were applied to search for differential metabolites. The metabolites were considered as potential altered metabolites according to the threshold of fold change $>1.2$ as well as $P<0.05$.

\section{RESULTS}

\section{Quality Evaluation of ZT by UHPLC-Q Exactive-Orbitrap-MS}

The components in ZT were extracted and analyzed by UHPLCQ Exactive-Orbitrap-MS and the total ion chromatogram 

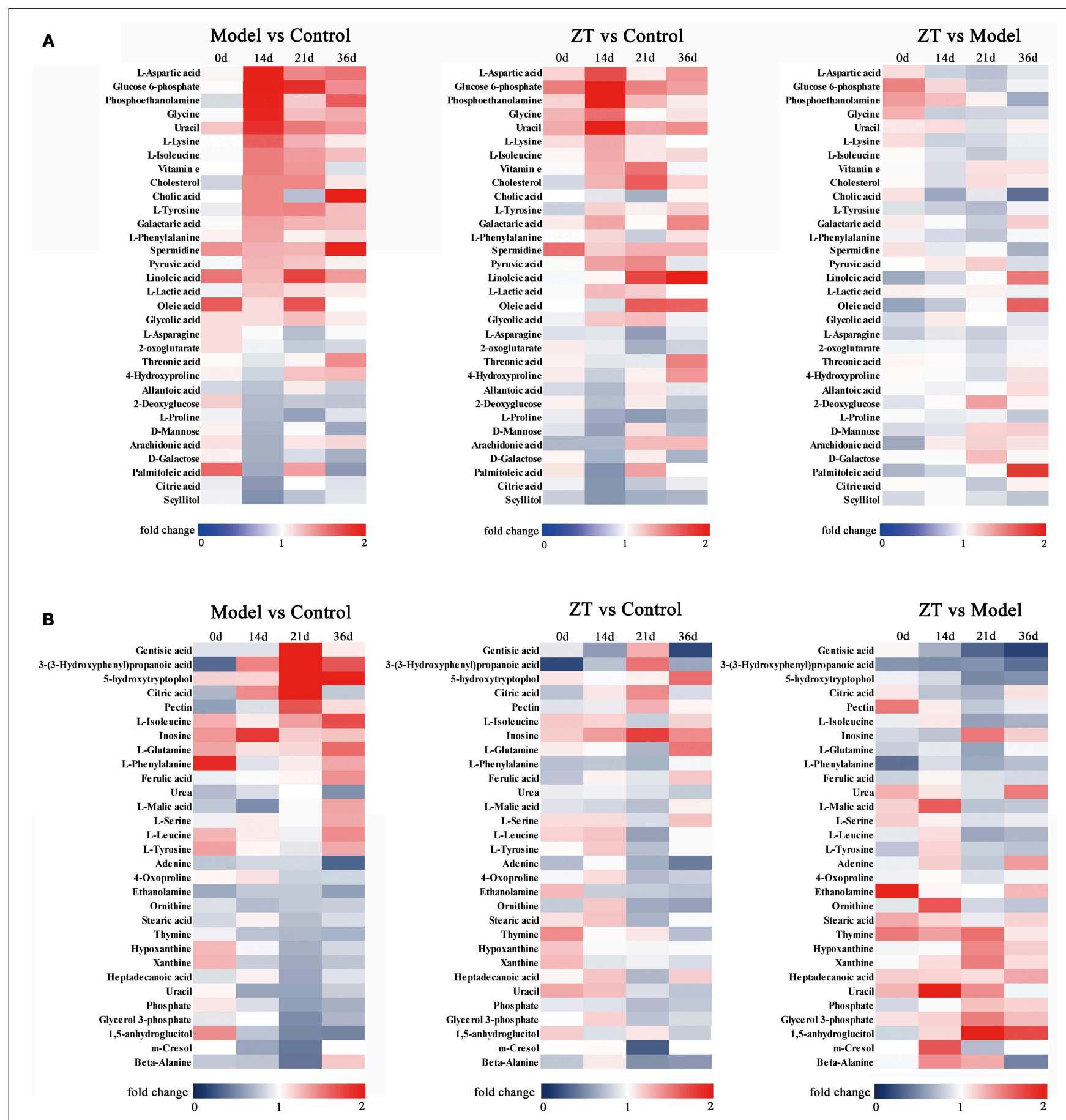

FIGURE 3 | Heatmap of identified differential metabolites with serum (A) and fecal (B) metabolomics profile. Each cell in the heatmap represents the fold change of a particular metabolite.

(TIC) of ZT and six standards is shown in Figures 1A,B, respectively. Another 18 components in ZT were identified, and the results are summarized in Table $\mathbf{1}$. In addition, daphnetin, 7hydroxycoumarin, and daphnoretin, three active ingredients that were reported to have therapeutic effects against RA (Shan et al., 2018), were determined at 1223.8, 485.1, and $131.3 \mu \mathrm{g}$ in each tablet, respectively.

\section{Basic Physiological Parameters}

Most of the rats fell ill on day 11 after primary immunization and exhibited hind limb stiffness, reduced activity, and ankle and toe inflammation. Four of the twenty-four rats failed to develop the disease and the incidence of RA was up to $83.33 \%$. Body weight, foot swelling, and arthritis scores are shown in Figures 2A-C. During the experiments, the body weight of rats in the model 


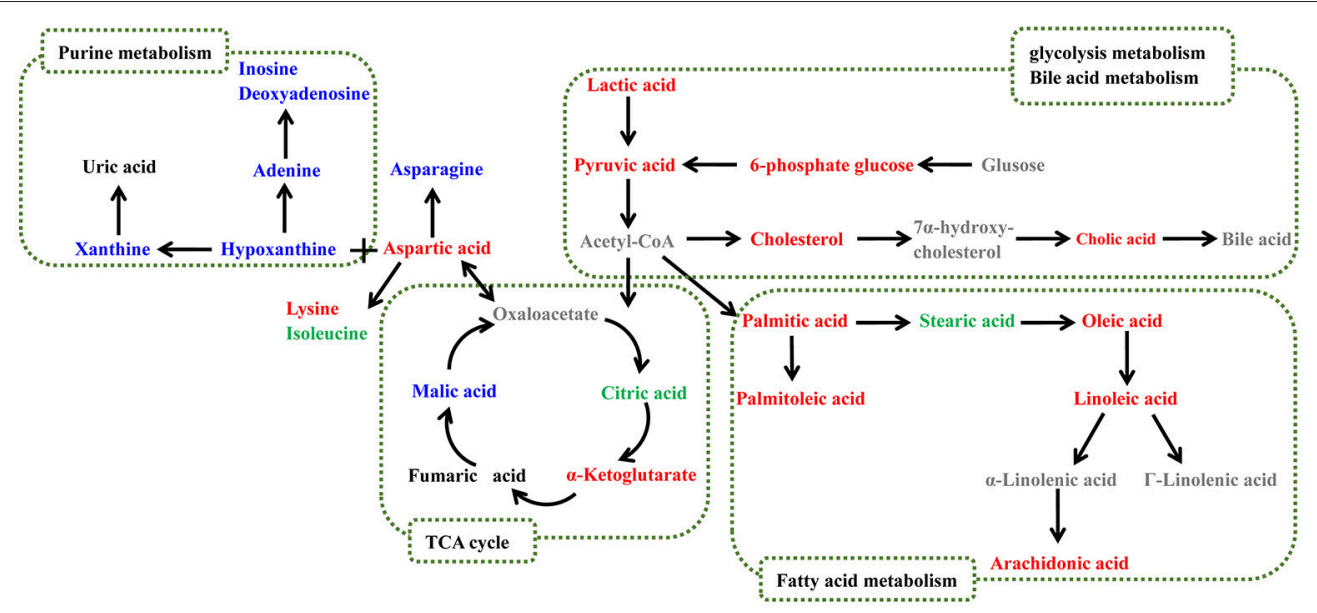

FIGURE 4 | Potential metabolic pathways disturbed in CIA rats. Metabolite names denoted in red or blue indicate that they were found in the serum or feces, respectively, and the metabolites shown in green were found in both. The names of the possible metabolic pathways are denoted in the green dotted box.

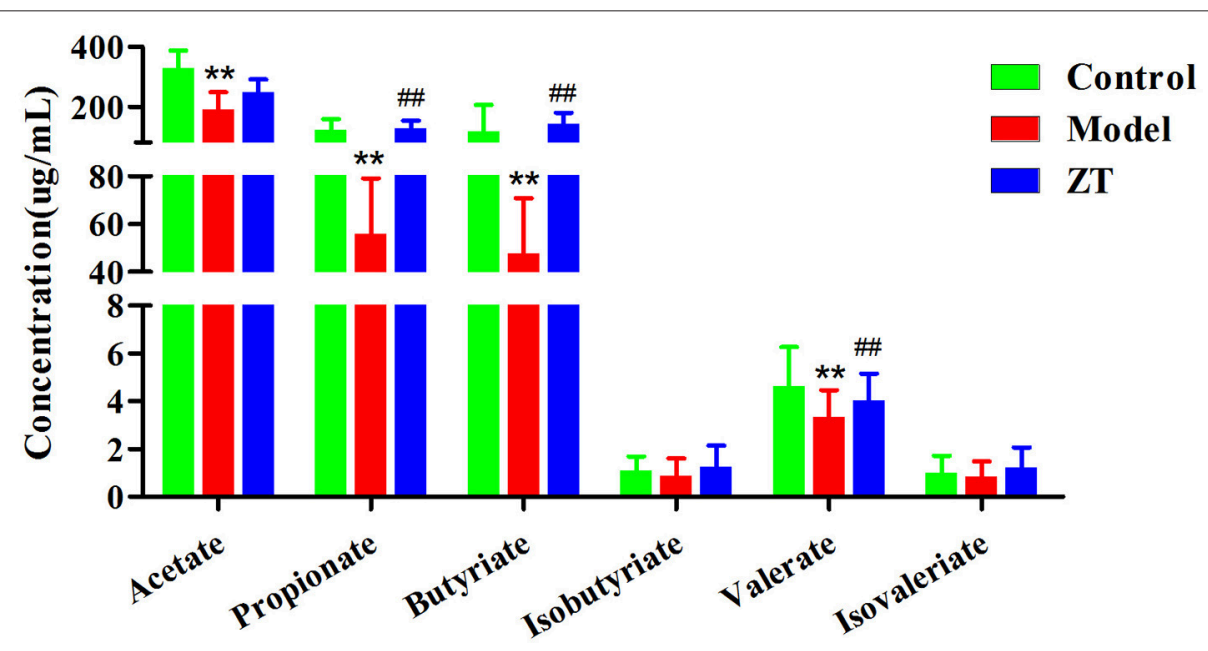

FIGURE 5 | The concentration of SCFAs in rat fecal samples in control, CIA rats, and ZT-treated rats. ${ }^{* *}$, vs. Control, $P<0.01$; \#\#, vs. Model, $P<0.01$.

and ZT groups increased slowly compared to that in the control group. The percentage of foot swelling in CIA rats increased rapidly on day 11 , and the swelling was attenuated by the oral administration of ZT. Arthritis scores also indicated that ZT demonstrated beneficial effects in CIA rats.

\section{Quantification of Serum TNF- $\alpha$ and IL-1 $\beta$ and histopathology Analysis}

Our study showed that serum IL-1 $\beta$ level was higher in the model group than in the control and ZT groups, while TNF- $\alpha$ did not exhibit any obvious difference among the three groups (Figure 2D). After hematoxylin-eosin (HE) staining, the synovial tissues of the ankle joint were observed under a microscope (Figures 2E-G). CIA rats exhibited obvious distinct synovial hyperplasia, and increased numbers of inflammatory cells, such as plasma cells, lymphocytes, and neutrophils, were present in the synovium. In the ZT group, inflammation was markedly attenuated and only few inflammatory cells were observed. In summary, the above results indicated that ZT could significantly inhibit inflammatory responses.

\section{System Stability and Reproducibility Analysis}

Both serum and fecal samples were analyzed based on GCMS to obtain biochemical information on metabolites. The GCMS TICs of serum and feces are shown in Figures S1A,B. To evaluate system stability and reproducibility, PCA analysis was performed to process the data matrix of QC samples. As shown in Figures S2A,B, in PCA score plots of serum and fecal samples, QC samples were clustered, which indicated that the stability of the GC-MS system was good throughout the whole analysis. In addition, the relative standard deviations (RSDs) of the peak 


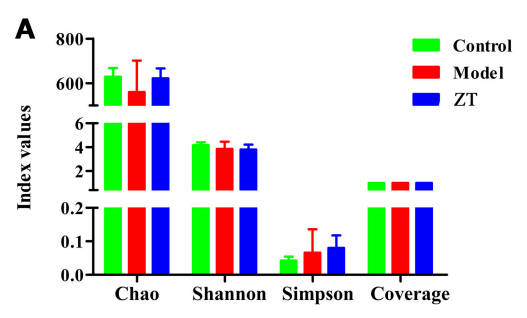

B

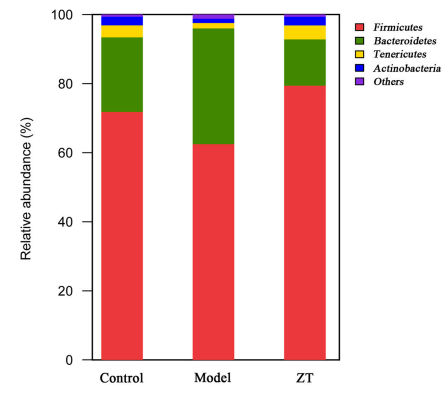

C

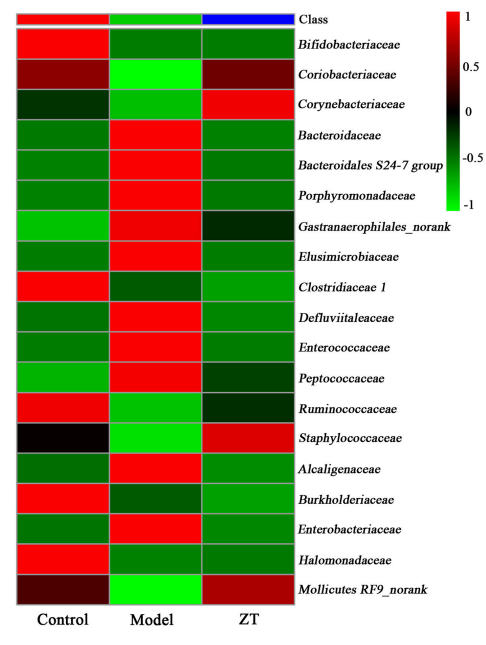

FIGURE 6 | The results of $16 \mathrm{~S}$ rRNA sequence analysis. (A) $\alpha$-diversity of chao, shannon, simpson, and coverage indices in control, CIA rats, and ZT-treated rats. (B) Gut microbiome composition profiles at the phylum level in control, CIA rats, and ZT-treated rats. (C) Microbial community of altered bacteria at the family level in control, CIA rats, and ZT-treated rats.

height of $1,2-{ }^{13} \mathrm{C}$-myristic acid was $15.39 \%$ for serum and $15.00 \%$ for feces, indicating that the analytical conditions and sample process exhibited good repeatability and stability for the metabolomics study.

\section{Differential Metabolites in Serum and Fecal Samples}

In serum samples, 95 endogenous metabolites were identified, including amino acids, glucose, and long-chain fatty acids. In addition, 99 endogenous metabolites were detected in fecal samples, including amino acids, amides, and pyrimidines. Fiftyone metabolites were detected in both the serum and feces, and these metabolites contained pyruvic acid, lactic acid, urea, and some amino acids.

The differential metabolites were selected based on one-way ANOVA and $\mathrm{q}$ test $(P<0.05)$, and there was a fold change $>1.2$ between the control and model groups on the same day. We filtered 31 potential biomarkers in the serum and 30 in the feces by comparing the model group with the control group. The detailed information about altered metabolites in the serum and fecal samples is presented in Tables 2, 3, respectively. To illustrate the fluctuations in altered serum and fecal metabolites and the content difference among the three groups, metabolic heatmaps of serum and feces were also generated (Figures 3A,B).

\section{Metabolic Pathway Analysis}

Biological pathway analysis was performed based on MetaboAnalyst 4.0, Kyoto Encyclopedia of Genes and Genomes (KEGG) (http://www.kegg.jp/), and Human Metabolome Database (HMDB) (http://www.hmdb.ca/). The main biochemical pathways related to RA included the TCA cycle, glycolysis metabolism, fatty acid metabolism, and purine metabolism. The metabolic pathway map is shown in Figure 4.

\section{Quantification of SCFAs in Fecal Samples}

As shown in Figure 5, acetate, propionate, and butyrate were the three most abundant SCFAs in rat feces. Compared with the control, the concentrations of all SCFAs decreased in CIA rats, especially acetate, propionate, butyrate, and valerate $(P<0.01)$, which indicated that the gut microbiota was disturbed under the influence of RA. The results also showed that $\mathrm{ZT}$ could regulate the content of propionate, butyrate, and valerate in the feces.

\section{Diversity Analysis of Gut Microbiota in Fecal Samples}

Based on fecal metabolomics and SCFA results, we speculated that RA might cause gut microbiota disorder. Therefore, gut microbiota diversity analysis was further performed to confirm the changes in gut microbiota and to discover the bacteria responsible for the altered metabolic profile of fecal samples. Diversity analysis, including $\alpha$-diversity and community structure, was performed based on the UPARSE software package. $\alpha$-diversity usually reflects the abundance of gut microbiota, and the microbial community structure is used to observe the community structure in different groups at the taxonomic level (phylum, family, and genus). Figure 6A shows four indices: Chao, Shannon, Simpson, and Coverage in different groups. The results indicate that $\alpha$-diversities of the gut microbiome in different groups did not show significant differences. Figure 6B shows the identified gut microbiota assigned at the phylum level, with each color representing an individual bacterial phylum. The results showed that Firmicutes (62.5-79.4\%) and Bacteroidetes (13.4-21.6\%) were the 
TABLE 4 | The $r$-value of correlation analysis for perturbed gut bacteria families and altered serum metabolites on day $36\left({ }^{\star} P<0.05 ;{ }^{\star \star} P<0.01\right)$.

\begin{tabular}{|c|c|c|c|c|c|}
\hline & Cholic acid & D-mannose & Palmitoleic acid & Spermidine & Threonic acid \\
\hline Bifidobacteriaceae & -0.205 & $0.463^{\star}$ & 0.208 & -0.344 & $-0.491^{\star \star}$ \\
\hline Coriobacteriaceae & $-0.411^{\star}$ & $0.446^{\star}$ & 0.154 & $-0.538^{\star \star}$ & -0.155 \\
\hline Corynebacteriaceae & $-0.511^{\star \star}$ & 0.287 & 0.285 & $-0.466^{\star \star}$ & -0.079 \\
\hline Bacteroidaceae & $0.565^{\star \star}$ & $-0.6^{\star \star}$ & -0.317 & $0.392^{\star}$ & 0.083 \\
\hline Bacteroidales S24-7 group & $0.411^{\star}$ & $-0.411^{\star}$ & $-0.365^{\star}$ & 0.257 & -0.093 \\
\hline Porphyromonadaceae & $0.447^{\star}$ & $-0.593^{\star \star}$ & $-0.38^{\star}$ & $0.407^{\star}$ & 0.073 \\
\hline Gastranaerophilales_norank & $0.578^{\star \star}$ & $-0.406^{\star}$ & $-0.369^{\star}$ & $0.422^{\star}$ & 0.216 \\
\hline Elusimicrobiaceae & 0.154 & -0.134 & -0.155 & $0.418^{*}$ & -0.038 \\
\hline Clostridiaceae 1 & -0.012 & 0.259 & 0.051 & -0.243 & $-0.408^{\star}$ \\
\hline Defluviitaleaceae & $0.462^{\star}$ & -0.026 & $-0.526^{\star \star}$ & $0.528^{\star \star}$ & 0.274 \\
\hline Enterococcaceae & $0.409^{\star}$ & -0.361 & $-0.468^{\star \star}$ & $0.487^{\star \star}$ & 0.021 \\
\hline Peptococcaceae & 0.306 & -0.164 & -0.322 & 0.16 & 0.241 \\
\hline Ruminococcaceae & $-0.481^{\star \star}$ & $0.386^{\star}$ & 0.158 & $-0.643^{\star \star}$ & -0.321 \\
\hline Staphylococcaceae & $-0.519^{\star \star}$ & 0.333 & $0.459^{\star}$ & $-0.629^{\star}$ & -0.243 \\
\hline Alcaligenaceae & $0.395^{\star}$ & $-0.417^{\star}$ & -0.357 & $0.417^{*}$ & 0.214 \\
\hline Burkholderiaceae & 0.004 & 0.147 & 0.059 & $-0.421^{*}$ & -0.266 \\
\hline Enterobacteriaceae & $0.515^{\star \star}$ & -0.261 & $-0.471^{\star \star}$ & $0.586^{\star \star}$ & 0.077 \\
\hline Halomonadaceae & -0.088 & $0.482^{\star \star}$ & 0.346 & -0.355 & $-0.385^{\star}$ \\
\hline Mollicutes RF9_norank & $-0.529^{\star \star}$ & 0.303 & 0.178 & $-0.499^{\star \star}$ & -0.19 \\
\hline
\end{tabular}

primary bacteria in the gut microbiota of SD rats, followed by Tenericutes (1.6-3.5\%) and Actinobacteria (1.3-2.5\%), while only Actinobacteria exhibited an obvious difference between the control and model groups $(P<0.05)$. Therefore, we decided to explore the difference at the family level between control and RA rats. Finally, we found that the content of 19 bacteria at the family level changed in model rats compared with controls, and the heatmap of these bacteria among three groups is shown in Figure 6C. In general, most of these changed bacteria belonged to the Actinobacteria, Bacteroidetes, Firmicutes, and Proteobacteria phylum while ZT showed a regulatory effect on Coriobacteriaceae, Bacteroidaceae, Porphyromonadaceae, among others.

\section{DISCUSSION}

\section{Pathway Analysis of Serum Metabolomics}

The identified serum biomarkers included 1-phenylalanine, 1aspartic acid, citric acid, glucose 6-phosphate, cholic acid, and cholesterol. It has been reported that these metabolites are related to the TCA cycle, glycolysis metabolism, and fatty acid metabolism.

\section{TCA Cycle and Glycolysis Metabolism}

The TCA cycle, which takes place in the mitochondria, is a key link in the metabolism of carbohydrates, lipids, and amino acids. The intermediates of the TCA cycle are also precursors of many biosynthetic pathways (Deng et al., 2016). The rate of the TCA cycle reflects the condition of energy metabolism. In this paper, the levels of citric acid decreased in the model group compared with the control group, indicating insufficient energy supply. In addition, it was reported that systemic inflammation induced by RA could lead to an increase in energy metabolism (Zhou et al., 2016). Within our identified altered metabolites, the level of l-isoleucine, an essential amino acid that can supply energy, was increased in CIA rats. When the rats were orally administered ZT, the serum level of 1isoleucine decreased, indicating that $\mathrm{ZT}$ was able to modulate energy metabolism.

Another metabolic pathway related to energy metabolism is glycolysis metabolism, which plays a key role in supplying ATP. In our study, the levels of pyruvate, lactic acid, and glucose 6-phosphate markedly increased during disease progression, indicating that the activity of glycolysis was enhanced.

Pyruvate could stimulate the transcription of vascular endothelial growth factor (VEGF) mRNA (Lee et al., 2001). In our previous study (Zhang et al., 2014), we found that the level of VEGF in RA synovial tissue increased significantly, and ZT decreased these levels, indicating that ZT could reduce the symptoms of RA by modulating the content of pyruvate. In addition, degradation of proline, which is associated with the TCA and urea cycle, was proven to fulfill the demand of ATP (Pandhare et al., 2009).

Smolenska et al. (2016) investigated the plasma concentrations of amino acid and nicotinamide metabolites in patients with RA and found that the level of 1-aspartic acid and 1-phenylalanine increased, while that of l-asparagine decreased in patients with RA. These results were confirmed in our experiments. Moreover, ZT markedly modulated the level of 1-aspartic acid and 1phenylalanine. RA was reported to cause dysfunction of protein synthesis and affect amino acid metabolism (Shin et al., 2011). 
TABLE 5 | The $r$-value of correlation analysis of perturbed gut bacteria families and altered fecal metabolites on day $36\left({ }^{\star} P<0.05 ;{ }^{\star \star} P<0.01\right)$.

\begin{tabular}{|c|c|c|c|c|c|c|c|c|c|c|}
\hline & Adenine & Ethanolamine & $\begin{array}{l}\text { Ferulic } \\
\text { acid }\end{array}$ & Glutamine & $\begin{array}{l}\text { L- } \\
\text { Isoleucine }\end{array}$ & $\begin{array}{l}\text { L- } \\
\text { e Leucine }\end{array}$ & $\begin{array}{l}\text { L- } \\
\text { Phenylalanine }\end{array}$ & $\begin{array}{l}\text { L- } \\
\text { Serine }\end{array}$ & Thymine & $\begin{array}{l}\text { L- } \\
\text { Tyrosine }\end{array}$ \\
\hline Bifidobacteriaceae & $0.393^{\star}$ & 0.307 & $-0.372^{\star}$ & -0.311 & -0.279 & -0.166 & -0.273 & $-0.381^{\star}$ & -0.017 & $-0.381^{\star}$ \\
\hline Coriobacteriaceae & 0.128 & 0.323 & $-0.419^{\star}$ & -0.151 & -0.19 & -0.188 & -0.147 & -0.084 & 0.267 & -0.188 \\
\hline Corynebacteriaceae & 0.245 & 0.324 & -0.053 & 0.087 & -0.09 & -0.002 & 0.093 & 0.152 & 0.372 & 0.046 \\
\hline Bacteroidaceae & -0.143 & -0.339 & 0.233 & 0.002 & 0.198 & 0.165 & 0.056 & -0.05 & -0.272 & 0.116 \\
\hline Bacteroidales S24-7 group & -0.209 & -0.324 & 0.158 & 0.036 & 0.209 & 0.179 & 0.092 & 0.014 & -0.188 & 0.122 \\
\hline Porphyromonadaceae & -0.127 & $-0.384^{\star}$ & 0.317 & 0.047 & 0.287 & 0.245 & 0.141 & 0.076 & -0.234 & 0.228 \\
\hline Gastranaerophilales_norank & -0.265 & $-0.475^{\star \star}$ & 0.305 & 0.289 & $0.399^{*}$ & 0.278 & 0.111 & 0.068 & $-0.459^{\star}$ & 0.12 \\
\hline Elusimicrobiaceae & -0.09 & $-0.452^{\star}$ & $0.437^{\star}$ & 0.072 & 0.021 & 0.107 & 0.017 & 0.105 & -0.046 & 0.118 \\
\hline Clostridiaceae 1 & 0.1 & 0.088 & $-0.443^{*}$ & -0.216 & -0.068 & 0.015 & 0.052 & -0.228 & 0.025 & 0.024 \\
\hline Defluviitaleaceae & -0.339 & -0.33 & 0.123 & 0.282 & $0.489^{\star}$ & $0.43^{\star}$ & $0.376^{\star}$ & 0.182 & -0.357 & 0.312 \\
\hline Enterococcaceae & -0.149 & $-0.407^{\star}$ & 0.274 & 0.099 & 0.257 & 0.304 & 0.262 & 0.154 & -0.12 & 0.286 \\
\hline Peptococcaceae & $-0.503^{\star \star}$ & -0.174 & -0.017 & 0.21 & 0.259 & 0.134 & 0.056 & 0.035 & -0.35 & 0.078 \\
\hline Ruminococcaceae & 0.327 & 0.279 & $-0.531^{\star *}$ & -0.319 & -0.294 & -0.265 & -0.155 & -0.24 & 0.104 & -0.257 \\
\hline Staphylococcaceae & $0.424^{\star}$ & $0.521^{\star \star}$ & -0.183 & 0.003 & -0.161 & -0.055 & 0.013 & 0.002 & $0.374^{\star}$ & -0.081 \\
\hline Alcaligenaceae & -0.219 & $-0.573^{\star \star}$ & 0.154 & -0.067 & 0.216 & 0.167 & 0.213 & 0.072 & -0.111 & 0.283 \\
\hline Burkholderiaceae & 0.295 & 0.172 & $-0.394^{\star}$ & -0.216 & -0.158 & -0.072 & -0.049 & -0.132 & 0.132 & -0.114 \\
\hline Enterobacteriaceae & -0.236 & $-0.414^{\star}$ & 0.223 & 0.072 & 0.294 & 0.235 & 0.111 & 0.074 & -0.241 & 0.158 \\
\hline Halomonadaceae & 0.255 & 0.269 & $-0.535^{\star \star}$ & $-0.403^{\star}$ & $-0.401^{\star}$ & -0.292 & -0.229 & -0.36 & 0.264 & -0.274 \\
\hline Mollicutes RF9_norank & 0.215 & 0.316 & -0.336 & -0.107 & -0.201 & -0.164 & -0.043 & -0.095 & 0.111 & -0.121 \\
\hline
\end{tabular}

\section{Fatty Acid Metabolism}

Another pathway related to RA is fatty acid metabolism. Among the differential metabolites in serum samples, palmitoleic acid, oleic acid, linoleic acid, and arachidonic acid were reported to participate in fatty acid metabolism. Fatty acids could also supply energy and serve as important sources of various lipid species ( Van Meer et al., 2008). Unsaturated fatty acids can promote the production of inflammatory cytokines TNF- $\alpha$ and IL- $1 \beta$, and then increase the production of PGE2 and NO, the two important inflammatory factors related to RA (Bruusgaard and Andersen, 1975; Ralston and Grabowski, 1996). In our investigation, ZT regulated the content level of palmitoleic acid markedly.

In accordance with our previous findings (Peng et al., 2017), markedly upregulated metabolites (linoleic acid and oleic acid) in fatty acid metabolism were observed in this investigation. The levels of both cholesterol and cholic acid were higher in RA rats than in the controls. The accumulation of cholesterol could promote inflammatory responses, inflammasome activation, and the production of monocytes as well as neutrophils.

\section{Pathway Analysis of Fecal Metabolomics}

The metabolomics study of feces was implemented to observe the altered metabolic profiles of gut microbiota and host cometabolites. Fecal metabolomics presented different results from those of the serum. As shown in Table 3, our identified biomarkers included l-tyrosine, $\mathrm{m}$-cresol, and nucleotide metabolites such as uracil, hypoxanthine, and thymine, which have been reported as gut microbiota-related metabolites (Zheng et al., 2013). The results suggested that the gut microbiota play an important role in RA-induced metabolic changes. According to the fecal metabolomics results, the important pathway related to RA was purine metabolism.

\section{Purine Metabolism}

It was reported that the level of uric acid increased in patients with RA, and excessive uric acid was deposited in the joints, soft tissues, and cartilage (Khondker and Khan, 2014; Liu et al., 2016). In our experiments, uric acid and its downstream products hypoxanthine, xanthine, and inosine were all detected, and they were all listed as biomarkers in fecal samples, indicating that RA had markedly affected purine metabolism. Inosine is an intermediate in the degradation of purines, while hypoxanthine is a naturally occurring purine derivative and a reaction intermediate in adenosine metabolism.

As reported previously (Nakajima et al., 2014), reactive oxygen species (ROS) were involved in the pathobiology of RA, and uric acid, the final product of purine and pyrimidine, could get eliminate ROS. Oxidative stress has also been implicated in the pathogenesis of RA (Charles-Schoeman et al., 2017). In addition, it was reported that daphnetin in $\mathrm{ZT}$ could reduce lipopolysaccharide (LPS)-induced production of ROS, indicating that ZT would exhibit anti-inflammatory effects probably by regulating the level of purine (Shen et al., 2017).

\section{The Role of SCFAs in Collagen-Induced Arthritis (CIA)}

It was reported that the severity of type II CIA was decreased after oral administration of either acetate, propionate, or butyrate (Mizuno et al., 2017), which indicated the significant role of SCFAs in the regulation of inflammation. This conclusion could also be confirmed in our experiment because the concentrations 
TABLE 6 | The $r$-value of correlation analysis of perturbed gut bacteria families and short chain fatty acids on day $36\left({ }^{\star} P<0.05\right.$; $\left.{ }^{\star \star} P<0.01\right)$.

\begin{tabular}{|c|c|c|c|c|c|c|}
\hline & Acetate & Propionate & Butyrate & Isobutyrate & Valerate & Isovalerate \\
\hline Bifidobacteriaceae & $0.667^{\star \star}$ & 0.352 & 0.144 & 0.1 & 0.333 & 0.161 \\
\hline Coriobacteriaceae & $0.404^{\star}$ & $0.556^{\star \star}$ & $0.414^{*}$ & 0.344 & $0.411^{\star}$ & 0.356 \\
\hline Corynebacteriaceae & 0.229 & 0.264 & $0.446^{\star}$ & 0.296 & 0.218 & 0.223 \\
\hline Bacteroidaceae & -0.332 & $-0.444^{\star}$ & -0.343 & -0.231 & -0.33 & -0.25 \\
\hline Bacteroidales S24-7 group & $-0.457^{\star}$ & $-0.459^{\star}$ & $-0.413^{\star}$ & -0.348 & $-0.477^{\star \star}$ & -0.304 \\
\hline Porphyromonadaceae & $-0.373^{\star}$ & $-0.403^{\star}$ & -0.348 & -0.315 & $-0.412^{\star}$ & -0.335 \\
\hline Gastranaerophilales_norank & $-0.45^{\star}$ & $-0.373^{\star}$ & -0.207 & 0.014 & -0.14 & 0.073 \\
\hline Elusimicrobiaceae & -0.075 & -0.346 & $-0.391^{\star}$ & $-0.437^{\star}$ & $-0.481^{\star \star}$ & $-0.435^{\star}$ \\
\hline Clostridiaceae 1 & $0.427^{\star}$ & -0.003 & -0.251 & -0.076 & 0.017 & -0.047 \\
\hline Defluviitaleaceae & -0.36 & $-0.407^{\star}$ & -0.362 & -0.019 & -0.049 & 0.011 \\
\hline Enterococcaceae & $-0.452^{*}$ & $-0.551^{\star \star}$ & $-0.406^{\star}$ & -0.246 & -0.242 & -0.199 \\
\hline Peptococcaceae & $-0.404^{\star}$ & $-0.383^{\star}$ & -0.124 & 0.128 & -0.107 & 0.186 \\
\hline Ruminococcaceae & 0.304 & $0.398^{\star}$ & 0.307 & 0.177 & 0.258 & 0.174 \\
\hline Staphylococcaceae & $0.382^{\star}$ & $0.445^{\star}$ & $0.527^{\star \star}$ & 0.314 & 0.34 & 0.255 \\
\hline Alcaligenaceae & $-0.377^{\star}$ & $-0.546^{\star \star}$ & -0.295 & -0.075 & -0.208 & -0.094 \\
\hline Burkholderiaceae & $0.446^{\star}$ & 0.297 & 0.285 & 0.259 & $0.426^{\star}$ & 0.241 \\
\hline Enterobacteriaceae & -0.359 & $-0.523^{\star \star}$ & -0.336 & -0.176 & -0.14 & -0.119 \\
\hline Halomonadaceae & $0.659^{\star \star}$ & 0.282 & 0.072 & 0.111 & 0.349 & 0.141 \\
\hline Mollicutes RF9_norank & 0.237 & $0.445^{\star}$ & 0.304 & 0.169 & 0.207 & 0.18 \\
\hline
\end{tabular}

of propionate, butyrate, and valerate increased in the ZT group. A possible reason for this result maybe that SCFAs could inhibit the disease by increasing the number of Tregs, a $\mathrm{T}$ cell subset for maintaining the immune response and suppressing excessive immune response (Zhou et al., 2015).

\section{Correlation Between Gut Microbiota and Metabolites of Serum and Feces}

It was reported that RA is a typical complex polygenic disease mediated by the human leukocyte antigen (HLA) system, and genetic differences largely determine the human body's susceptibility to the disease (Cai et al., 2012). According to the US WebMD Medical News (Taneja, 2014), the link between the genetic predisposition to RA and environmental factors may depend on the gut microbiota. Several studies supported this point of view and indicated that the gut microbiota is crucial to maintain the balance of the immune system in patients with RA. Gomez et al. explored the interaction between genetic factors and gut microbiota, and demonstrated that altered gut microbiota could predict the body's susceptibility to RA (Gomez et al., 2012). Lactobacillus could significantly reduce serum TNF- $\alpha$, IL6 , and other proinflammatory cytokines in patients with RA (Li et al., 2015a). In our present study, the correlation between changed gut microbiota and serum and fecal biomarkers and SCFAs was analyzed based on Pearson's correlation coefficient, and the $r$-values are presented in Tables 4-6, while the $P$-values are shown in Tables S2-S4. The gut microbiota is closely related to the bile acid pool. Primary bile acids were synthesized in the liver from cholesterol (Chiang, 2009). The gut microbiota modifies the bile acid steroid core and transforms the primary bile acids to secondary bile acids. Along with the change in the gut microbiome between control and CIA rats, the bile acid pool was also disturbed. As shown in Table 4, cholic acid was highly correlated with the gut microbiome, including Bacteroidaceae, Bacteroidales S24-7 group, Enterococcaceae, and Corynebacteriaceae.

It was also observed (Table 5) that the Defluviitaleaceae family was positively and highly correlated with l-isoleucine, 1-leucine, and 1-phenylalanine while Defluviitaleaceae was reported to regulate the metabolism of amino acids, energy, and carbohydrates (Zhao et al., 2018). Halomonadaceae was negatively correlated with ferulic acid, l-glutamine, and 1isoleucine in our research.

As shown in Table 6, acetate, propionate, butyrate, and valerate showed obvious correlation with almost all species, and the Bacteroidales S24-7 group in particular. The Bacteroidales S24-7 group, also named Candidatus Homeothermaceae, is capable of producing acetate, propionate, and succinate (Ormerod et al., 2016). However, in our research, it was negatively correlated with all SCFAs except isobutyrate and isovalerate, and further exploration is needed to determine the reason.

To the best of our knowledge, this is the first study to evaluate the therapeutic effects of ZT on RA by metabolomics and gut microbiota analysis. However, our study has several limitations: (1) We only detected small molecules with $\mathrm{m} / \mathrm{z}$ values under 500 using the GC-MS method, so it was difficult to comprehensively understand the overall metabolic process of RA. In future studies, we will apply the LC-MS technique to comprehensively monitor metabolic alterations in RA and evaluate the mechanism of ZT. (2) Our preliminary investigation found the correlation between gut microbiota and fecal metabolites, but additional experiments should be designed 
in future studies using special antibiotics administration to shift the composition of the gut microbiota and confirm the conclusion. (3) Our research was conducted based on rats; however, human samples should be collected from RA patients for metabolomics analysis to explore the biomarkers of the disease.

\section{CONCLUSION}

In this study, serum and fecal metabolomics based on GC-MS, as well as 16S rRNA sequence analysis and pharmacodynamic evaluation (weight, foot swelling, arthritis score, inflammatory factor, and histopathology) were applied to determine the therapeutic effects of ZT on RA. The main objective of this study was to reveal the metabolic profiles of RA and the regulatory mechanisms of ZT. Several altered metabolites were identified, including l-asparagine, citric acid, l-isoleucine, and hypoxanthine. Furthermore, the pathway analysis of serum metabolomics demonstrated that the TCA cycle and fatty acid metabolism were disrupted in CIA rats. Disorders in purine metabolism were observed from the results of fecal metabolomics analysis. Sequence analysis results showed that the composition of gut microbiota was disturbed in CIA rats at the level of the family, and this could be rectified to some extent by ZT. In summary, this work indicated that metabolomics is a systematic approach for evaluating the therapeutic effects and mechanism of ZT.

\section{REFERENCES}

Arend, W. P., and Dayer, J. M. (1995). Inhibition of the production and effects of interleukin-1 and tumor necrosis factor alpha in rheumatoid arthritis. Arthritis Rheum. 38, 151-160. doi: 10.1002/art.1780380202

Bruusgaard, A., and Andersen, R. B. (1975). Effect of an intravenously administered bile acid (chenodeoxycholic acid) on rheumatoid arthritis. Scand. J. Rheumatol. 4, 169-173. doi: 10.3109/03009747509165448

Cai, W. H., Sun, B. D., Zhang, B. F., Yue, Y., Cheng, W. X., Hu, Y. P., et al. (2012). Overview of the etiology of rheumatoid arthritis. China Mod. Med. 19, 11-12.

Cajka, T., Smilowitz, J. T., and Fiehn, O. (2017). Validating quantitative untargeted lipidomics across nine liquid chromatography-highresolution mass spectrometry platforms. Anal. Chem. 89, 12360-12368. doi: 10.1021/acs.analchem.7b03404

Cavagna, L., Caporali, R., Trifiro, G., Arcoraci, V., Rossi, S., and Montecucco, C. (2013). Overuse of prescription and OTC non-steroidal anti-inflammatory drugs in patients with rheumatoid arthritis and osteoarthritis. Int. J. Immunopathol. Pharmacol. 26, 279-281. doi: 10.1177/039463201302600132

Charles-Schoeman, C., Meriwether, D., Lee, Y. Y., Shahbazian, A., and Reddy, S. T. (2017). High levels of oxidized fatty acids in HDL are associated with impaired HDL function in patients with active rheumatoid arthritis. Clin. Rheumatol. 37, 615-622. doi: 10.1007/s10067-017-3896-y

Chen, J., Wright, K., Davis, J. M., Jeraldo, P., Marietta, E. V., Murray, J., et al. (2016). An expansion of rare lineage intestinal microbes characterizes rheumatoid arthritis. Genome Med. 8:43. doi: 10.1186/s13073-016-0299-7

Chen, L. T., Jing, Y. Y., Di, L. Q., Xu, H. Q., Wu, H., and Shan, J. J. (2011). Study on the Daphne giraldii Nitsche. effective parts of anti-rheumatoid arthritis. Res. Pract. Chin. Med. 25, 37-40. doi: 10.13728/j.1673-6427.2011.02.018

Chiang, J. Y. (2009). Bile acids: regulation of synthesis. J. Lipid Res. 50, 1955-1966. doi: 10.1194/jlr.R900010-JLR200

\section{ETHICS STATEMENT}

This study was carried out in accordance with the recommendations of Animal Ethics Committee of Nanjing University of Chinese Medicine. The protocol was approved by the Animal Ethics Committee of Nanjing University of Chinese Medicine.

\section{AUTHOR CONTRIBUTIONS}

LD and JS designed the experiments. LP, JS, WQ, and TX performed the experiments, analyzed the data and wrote the manuscript. AK and BG amended the paper.

\section{FUNDING}

This investigation was supported by the National Natural Science Foundation of China (No. 81273655). Postgraduate Research and Practice Innovation Program of Jiangsu Province (No. KYCX17_1323). Jiangsu Provincial 333 High-level Talents Cultivation Project and Jiangsu Provincial Six Talent Peaks Project.

\section{SUPPLEMENTARY MATERIAL}

The Supplementary Material for this article can be found online at: https://www.frontiersin.org/articles/10.3389/fphar. 2018.00891/full\#supplementary-material

Deng, L., Gu, H., Zhu, J., Nagana Gowda, G. A., Djukovic, D., Chiorean, E. G., et al. (2016). Combining NMR and LC/MS using backward variable elimination: metabolomics analysis of colorectal cancer, polyps, and healthy controls. Anal. Chem. 88, 7975-7983. doi: 10.1021/acs.analchem.6b00885

Gao, Q., Shan, J., Di, L., Jiang, L., and Xu, H. (2008). Therapeutic effects of daphnetin on adjuvant-induced arthritic rats. J. Ethnopharmacol. 120, 259-263. doi: 10.1016/j.jep.2008.08.031

Gomez, A., Luckey, D., Yeoman, C. J., Marietta, E. V., Berg Miller, M. E., Murray, J. A., et al. (2012). Loss of sex and age driven differences in the gut microbiome characterize arthritis-susceptible 0401 mice but not arthritis-resistant 0402 mice. PLoS ONE 7:e36095. doi: 10.1371/journal.pone.0036095

Gu, H., Zhang, P., Zhu, J., and Raftery, D. (2015). Globally optimized targeted mass spectrometry: reliable metabolomics analysis with broad coverage. Anal. Chem. 87, 12355-12362. doi: 10.1021/acs.analchem.5b03812

Huyiligeqi, Dong, X., Yang, C., Xu, G., Cao, S., Fu, J., et al. (2016). Chemical constituents from daphne giraldii nitsche and their contents simultaneous determination by HPLC. Evid. Based Complement. Alternat. Med. 2016:9492368. doi: 10.1155/2016/9492368

Khamis, M. M., Adamko, D. J., and El-Aneed, A. (2017). Mass spectrometric based approaches in urine metabolomics and biomarker discovery. Mass Spectrom. Rev. 36, 115-134. doi: 10.1002/mas.21455

Khondker, L., and Khan, S. I. (2014). Association of rheumatoid factor and uric acid with psoriatic arthritis: a review. Mymensingh Med. J. 23, 609-613.

Kundu, S., Ghosh, P., Datta, S., Ghosh, A., Chattopadhyay, S., and Chatterjee, M. (2012). Oxidative stress as a potential biomarker for determining disease activity in patients with rheumatoid arthritis. Free Radic. Res. 46, 1482-1489. doi: 10.3109/10715762.2012.727991

Lee, M. S., Moon, E. J., Lee, S. W., Kim, M. S., Kim, K. W., and Kim, Y. J. (2001). Angiogenic activity of pyruvic acid in in vivo and in vitro angiogenesis models. Cancer Res. 61, 3290-3293. 
Li, M., Sun, D., Fu, B. B., and Sun, X. P. (2015a). Research on gut microbiota and autoimmune disease: progress review. Chin. J. Microecol. 27, 1233-1237. doi: 10.13381/j.cnki.cjm.201510032

Li, Y., Wang, J., Xiao, Y., Wang, Y., Chen, S., Yang, Y., et al. (2015b). A systems pharmacology approach to investigate the mechanisms of action of Semen Strychni and Tripterygium wilfordii Hook F for treatment of rheumatoid arthritis. J. Ethnopharmacol. 175, 301-314. doi: 10.1016/j.jep.2015.09.016

Liu, S., Li, H. Y., and Zhao, L. (2016). Research progress on lowering uric acid and anti-inflammation effect of resveratrol. Drug Eval. Res. 39, 304-307. doi: 10.7501/j.issn.1674-6376.2016.02.032

Mizuno, M., Noto, D., Kaga, N., Chiba, A., and Miyake, S. (2017). The dual role of short fatty acid chains in the pathogenesis of autoimmune disease models. PLoS ONE 12:e0173032. doi: 10.1371/journal.pone.0173032

Moller, B., Pruijm, M., Adler, S., Scherer, A., Villiger, P. M., Finckh, A., et al. (2015). Chronic NSAID use and long-term decline of renal function in a prospective rheumatoid arthritis cohort study. Ann. Rheum. Dis. 74, 718-723. doi: 10.1136/annrheumdis-2013-204078

Nakajima, A., Aoki, Y., Shibata, Y., Sonobe, M., Terajima, F., Takahashi, H., et al. (2014). Identification of clinical parameters associated with serum oxidative stress in patients with rheumatoid arthritis. Mod. Rheumatol. 24, 926-930. doi: 10.3109/14397595.2014.891495

Ormerod, K. L., Wood, D. L., Lachner, N., Gellatly, S. L., Daly, J. N., Parsons, J. D., et al. (2016). Genomic characterization of the uncultured Bacteroidales family S24-7 inhabiting the guts of homeothermic animals. Microbiome 4:36. doi: 10.1186/s40168-016-0181-2

Pandhare, J., Donald, S. P., Cooper, S. K., and Phang, J. M. (2009). Regulation and function of proline oxidase under nutrient stress. J. Cell. Biochem. 107, 759-768. doi: $10.1002 /$ jcb. 22174

Peng, L. X., Chen, L. H., Di, L. Q., Shan, J. J., Xie, T., Kang, A., et al. (2017). Plasma metabonomic study on Zushima Gancao tablet in treatment of rheumatoid arthritis based on UPLC/LTQ-Orbitrap-MS. Chin. Tradit. Herbal Drugs 48, 1964-1970. doi: 10.7501/j.issn.0253-2670.2017.10.006

Qi, Y., Li, S., Pi, Z., Song, F., Lin, N., Liu, S., et al. (2014). Metabonomic study of Wu-tou decoction in adjuvant-induced arthritis rat using ultraperformance liquid chromatography coupled with quadrupole time-of-flight mass spectrometry. J. Chromatogr. B Analyt. Technol. Biomed. Life Sci. 953-954, 11-19. doi: 10.1016/j.jchromb.2014.01.044

Ralston, S. H., and Grabowski, P. S. (1996). Mechanisms of cytokine induced bone resorption: role of nitric oxide, cyclic guanosine monophosphate, and prostaglandins. Bone 19, 29-33. doi: 10.1016/8756-3282(96)00101-9

Shan, J., Qian, W., Peng, L., Chen, L., Kang, A., Xie, T., et al. (2018). A comparative pharmacokinetic study by UHPLC-MS/MS of main active compounds after oral administration of zushima-gancao extract in normal and adjuvant-induced arthritis rats. Molecules 23:e227. doi: 10.3390/molecules23010227

Shen, L., Zhou, T., Wang, J., Sang, X., Lan, L., Luo, L., et al. (2017). Daphnetin reduces endotoxin lethality in mice and decreases LPS-induced inflammation in Raw264.7 cells via suppressing JAK/STATs activation and ROS production. Inflamm. Res. 66, 579-589. doi: 10.1007/s00011-017-1039-1

Shin, J. H., Yang, J. Y., Jeon, B. Y., Yoon, Y. J., Cho, S. N., Kang, Y. H., et al. (2011). $1 \mathrm{H}$ NMR-based metabolomic profiling in mice infected with Mycobacterium tuberculosis. J. Proteome Res. 10, 2238-2247. doi: 10.1021/pr101054m

Smolenska, Z., Smolenski, R. T., and Zdrojewski, Z. (2016). Plasma concentrations of amino acid and nicotinamide metabolites in rheumatoid arthritis-potential biomarkers of disease activity and drug treatment. Biomarkers 21, 218-224. doi: 10.3109/1354750X.2015.1130746

Suzuki, T., Yoshida, S., and Hara, H. (2008). Physiological concentrations of shortchain fatty acids immediately suppress colonic epithelial permeability. Br. J. Nutr. 100, 297-305. doi: 10.1017/S0007114508888733

Tan, J., McKenzie, C., Potamitis, M., Thorburn, A. N., Mackay, C. R., and Macia, L. (2014). The role of short-chain fatty acids in health and disease. Adv. Immunol. 121, 91-119. doi: 10.1016/B978-0-12-800100-4.00003-9

Taneja, V. (2014). Arthritis susceptibility and the gut microbiome. FEBS Lett. 588, 4244-4249. doi: 10.1016/j.febslet.2014.05.034

Tsugawa, H., Cajka, T., Kind, T., Ma, Y., Higgins, B., Ikeda, K., et al (2015). MS-DIAL: data-independent MS/MS deconvolution for comprehensive metabolome analysis. Nat. Methods 12, 523-526. doi: 10.1038/nmeth.3393

Van Meer, G., Voelker, D. R., and Feigenson, G. W. (2008). Membrane lipids: where they are and how they behave. Nat. Rev. Mol. Cell Biol. 9, 112-124. doi: $10.1038 / \mathrm{nrm} 2330$

Van Vollenhoven, R. F. (2009). Treatment of rheumatoid arthritis: state of the art 2009. Nat. Rev. Rheumatol. 5, 531-541. doi: 10.1038/nrrheum.2009.182

Wu, X. M., and Li, Y. J. (2010). Experimental Methodology of Pharmacology. Beijing: People's Medical Publishing House.

Xi, B., Gu, H., Baniasadi, H., and Raftery, D. (2014). Statistical analysis and modeling of mass spectrometry-based metabolomics data. Methods Mol. Biol. 1198, 333-353. doi: 10.1007/978-1-4939-1258-2 22

Zhang, W., Gong, L., Zhou, L. L., Shan, J. J., Chen, L. T., Xu, H. Q., et al. (2014). Effect of different compatibility of Daphnes giraldii cortex and Glycyrrhizae radix et Rhizoma on adjuvant-induced arthritis in rats. Chin. Tradit. Herbal Drugs 45, 1418-1426. doi: 10.7501/j.issn.0253-2670.2014.10.013

Zhao, J., Yao, Y., Li, D., Xu, H., Wu, J., Wen, A., et al. (2018). Characterization of the Gut microbiota in six geographical populations of chinese Rhesus macaques (Macaca mulatta), implying an adaptation to high-altitude environment. Microb. Ecol. 76, 565-577 doi: 10.1007/s00248-018-1146-8

Zheng, X., Zhao, A., Xie, G., Chi, Y., Zhao, L., Li, H., et al. (2013). Melamineinduced renal toxicity is mediated by the gut microbiota. Sci. Transl. Med. 5:172ra122. doi: 10.1126/scitranslmed.3005114

Zhou, C., Qing, Y. F., and Zhou, J. G. (2015). Regulatory T cells and the research progress in autoimmune diseases. Shandong Med. J. 55, 96-98. doi: 10.3969/j.issn.1002- 266X.2015.24.044

Zhou, J., Chen, J., Hu, C., Xie, Z., Li, H., Wei, S., et al. (2016). Exploration of the serum metabolite signature in patients with rheumatoid arthritis using gas chromatography-mass spectrometry. J. Pharm. Biomed. Anal. 127, 60-67. doi: 10.1016/j.jpba.2016.02.004

Conflict of Interest Statement: The authors declare that the research was conducted in the absence of any commercial or financial relationships that could be construed as a potential conflict of interest.

Copyright (c) 2018 Shan, Peng, Qian, Xie, Kang, Gao and Di. This is an open-access article distributed under the terms of the Creative Commons Attribution License (CC $B Y)$. The use, distribution or reproduction in other forums is permitted, provided the original author(s) and the copyright owner(s) are credited and that the original publication in this journal is cited, in accordance with accepted academic practice. No use, distribution or reproduction is permitted which does not comply with these terms. 